\title{
ELECTROLYSIS AND STAINLESS STEELS IN BONE
}

\author{
J. K. Wright and H. J. Axon, Manchester, England
}

From the Departments of Orthopaedic Surgery and of Metallurgy, University of Manchester

There is still much mystery in the loosening of metal screws inserted in bone. Why do some loosen while others remain so fixed that they will break rather than rotate when the surgeon's screwdriver tries to remove them? There is, of course, no single answer to this question. Some screws loosen because they are inserted badly; others through faults in their metals; some from sepsis and some from other causes. In this paper we will discuss the part played by electrolysis in this loosening, a factor which has been brought to our attention once again by the work of Bowden, Williamson and Laing (1954). We do not propose to discuss the non-ferrous alloys (Vitallium, etc.) but to confine our attention to the more commonly used stainless steels.

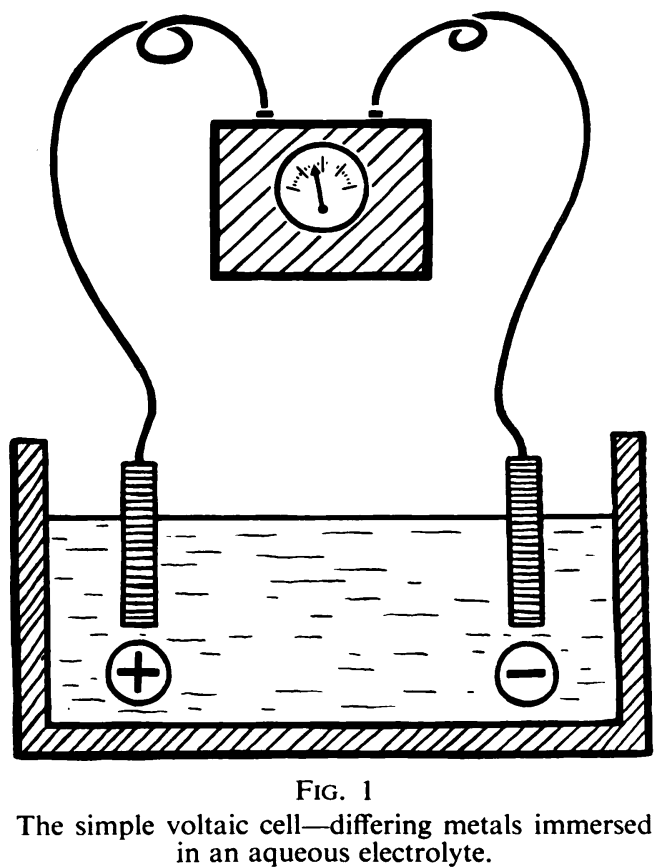

THE VOLTAIC CELL

If two different metals or alloys of different chemical composition are in electrical contact in the presence of an aqueous electrolyte, a voltaic cell is set up (Fig. 1), and an electric current will flow from one piece of metal, through the electrolyte, to the other. As regards the flow of positive electricity, the piece of metal from which the current flows into the electrolyte is designated the anode, and suffers electrolytic dissolution or corrosion. The other piece of metal, into which the current flows from the electrolyte, is designated the cathode, and receives a deposition of hydrogen ions or metal ions. If oxygen is present on the cathode it may combine with the discharged hydrogen ions to form water.

If the anode is a screw inserted into bone, living or dead, corrosion will cause it to loosen. This is due partly to the loss of metal from its surface, but much more to the superficial disappearance of the bone with which it is in contact. The loss of bone may be due to its

VOL. 38 B, NO. 3, AUGUST 1956 
active involvement in the electrolytic process, but it is more likely that the bone is attacked by the products of electrolysis as a purely chemical phenomenon. If these changes occur in living tissue the gap left by loss of bone is filled with fibrous tissue in which small metallic particles are embedded (Fig. 2). This fibrous tissue, though tough, has not the strength of

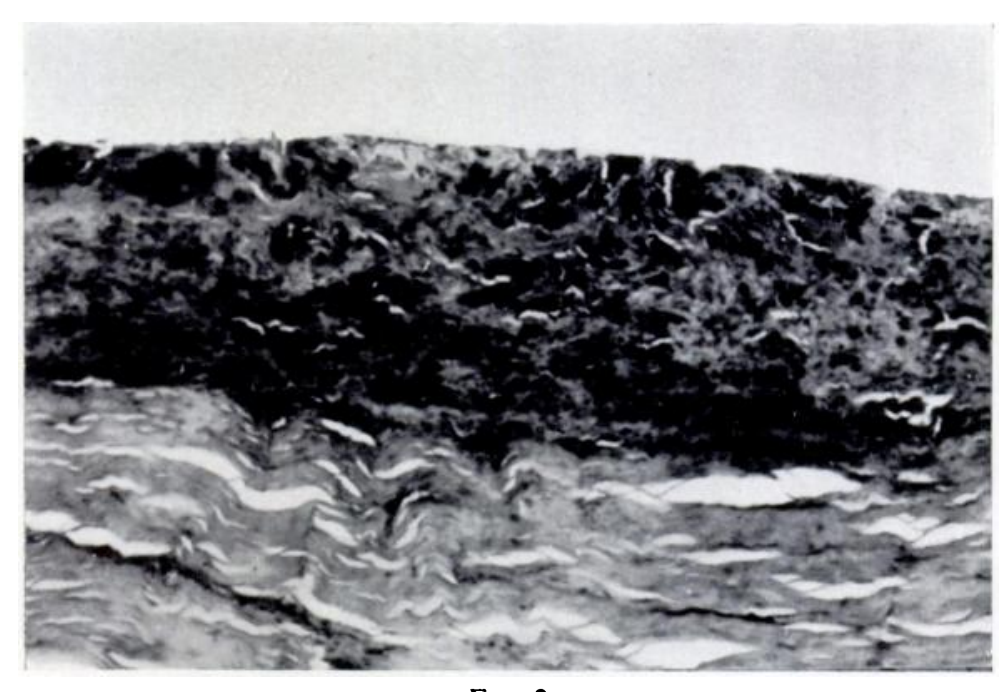

Fig. 2

Metallic particles in fibrous tissue near a corroded screw. (Prussian blue, $\because 110$.

bone, and if the screw is under considerable mechanical strain will fail to hold it. Once any movement of the screw is possible the "play" is gradually increased mechanically, as occurs when one removes a nail from a piece of wood by repeatedly bending it in opposite directions. The electrolytic bony dissolution produces a shallow, conical defect which can be seen in a radiograph around one or other extremity of the screw (Fig. 3). Movement produces a

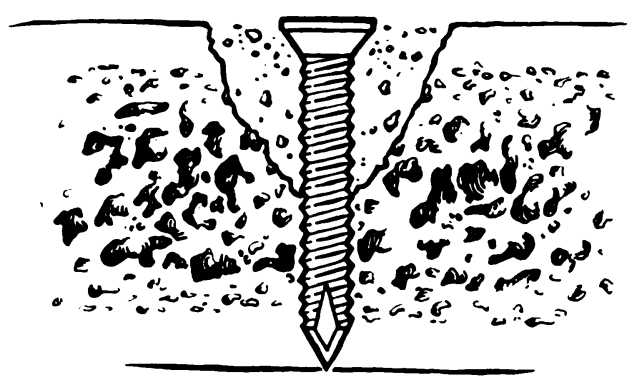

Fig. 3

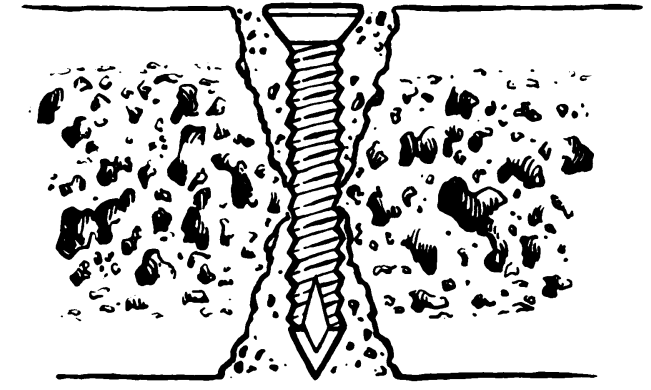

Fig. 4

Figure 3-Pattern of bony erosion due to electrolysis. Figure 4-Pattern of bony erosion due to movement of the screw.

narrower conical defect visible at one or both ends (Fig. 4). Such movement predisposes to the fracture of the screw at the fulcrum when the bone it is securing is "shock-loaded" by a sudden strain or fall. This is more likely to occur if the screw is also locally corroded.

If the electrolytic process is speeded up by passing an electric current from a battery to two screws inserted into dead bone and immersed in an electrolyte such as normal saline or plasma, changes can be produced identical with those occurring in vivo. Figures 5 to 7 show the result of such an experiment. The stainless steel screw shows extensive destruction, and the surrounding bone is removed. It is interesting to note that bone is here being removed 
by an electro-chemical process and not by osteoclasis. Whether or not this can occur in vivo is unknown, but the similarity of radiological appearance in both cases suggests that it is possible.

The amount of corrosive attack depends on the amount of current per unit of time

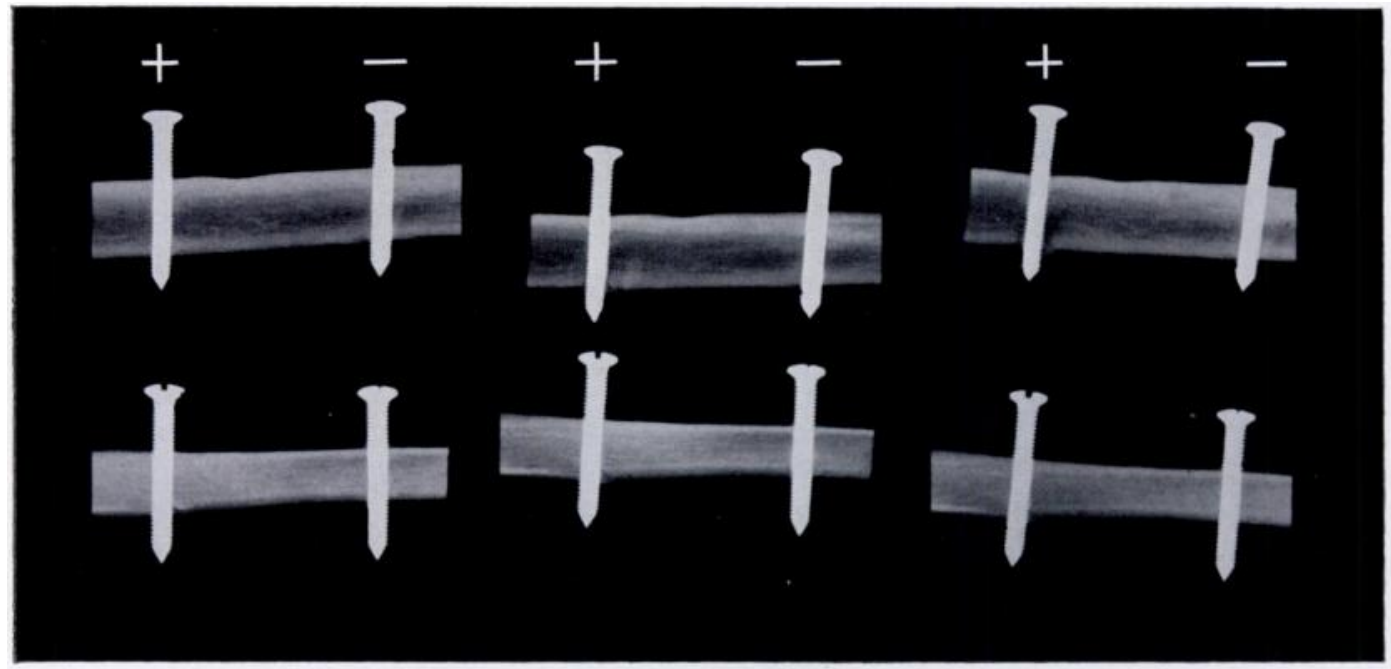

FiG. 5

Radiographic appearances at 0,20 and 40 hours after passage of 10 milliamp current, with bone and screws immersed in bath of normal saline. (Control below.)

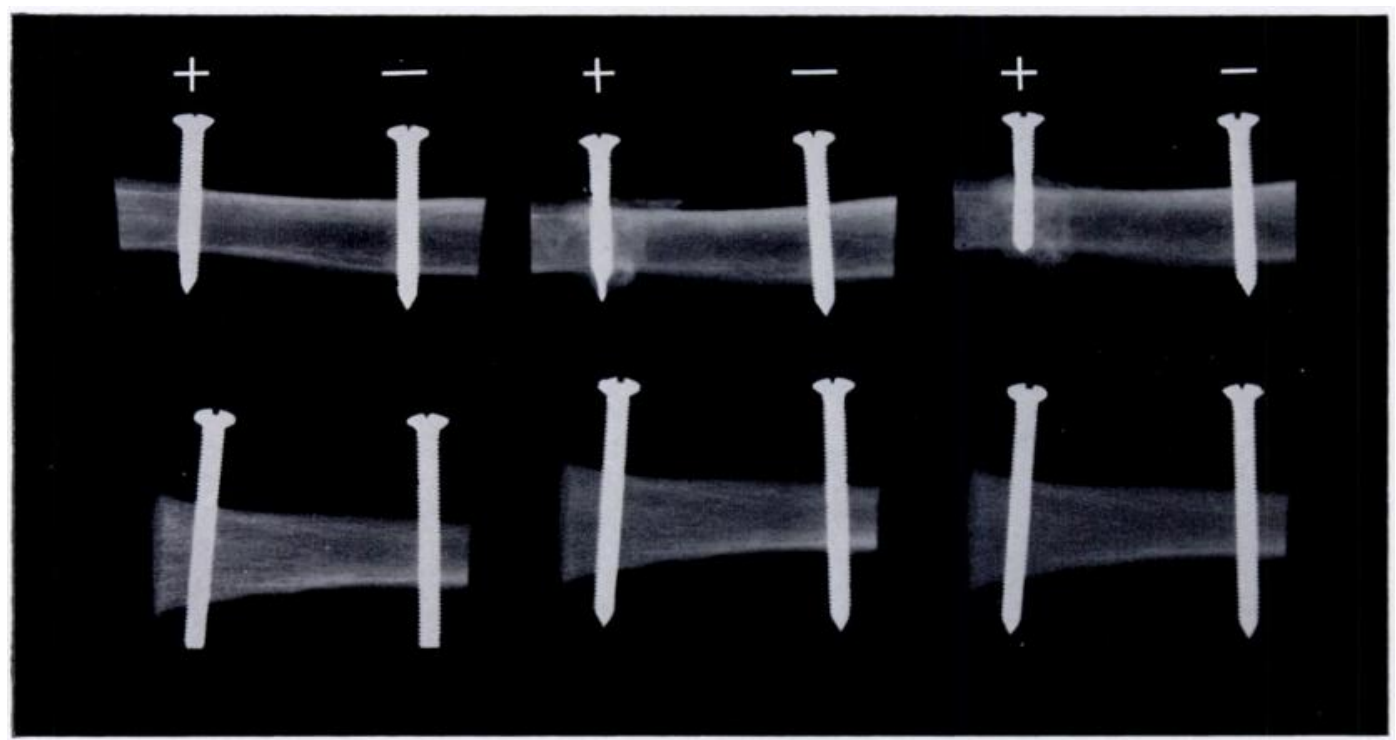

FiG. 6

Similar experiment with bonei mmersed in human plasma. Marked corrosion of stainless steel screw.

passing through the voltaic circuit and hence upon the electrical conductivity of the circuit. In isolated screws in bone (as in an onlay graft) the current flowing depends upon the conductivity of the tissue fluids between them. If the screws are joined electrically by a plate between them the conductivity of the circuit is increased, and consequently more corrosion can be expected to occur.

VOL. 38 B, NO. 3, AUGUST 1956 
It is precisely to prevent the above phenomena that surgeons use stainless steel materials in the body, as these, which have been presumed to be of uniform composition, are regarded (Venable and Stuck 1947) as less electrically active. Yet screws of stainless steel do loosen in bone. Can such loosening be due to electrolysis, and, if so, how important is this factor as a cause of non-union of fractures treated by internal fixation with screws and plates of stainless steel?

\section{FACTORS INFLUENCING THE CORROSION OF STAINLESS STEEL SCREWS}

There are five important factors that may lead to corrosion of stainless steels and to loosening of screws composed of it. They are 1) chemical composition of components; 2) surface abrasion; 3) cold welding; 4) mechanical distortion; 5) differential oxygenation.

Chemical composition of components-All "stainless steels" are not of the same chemical composition; there are, indeed, many different

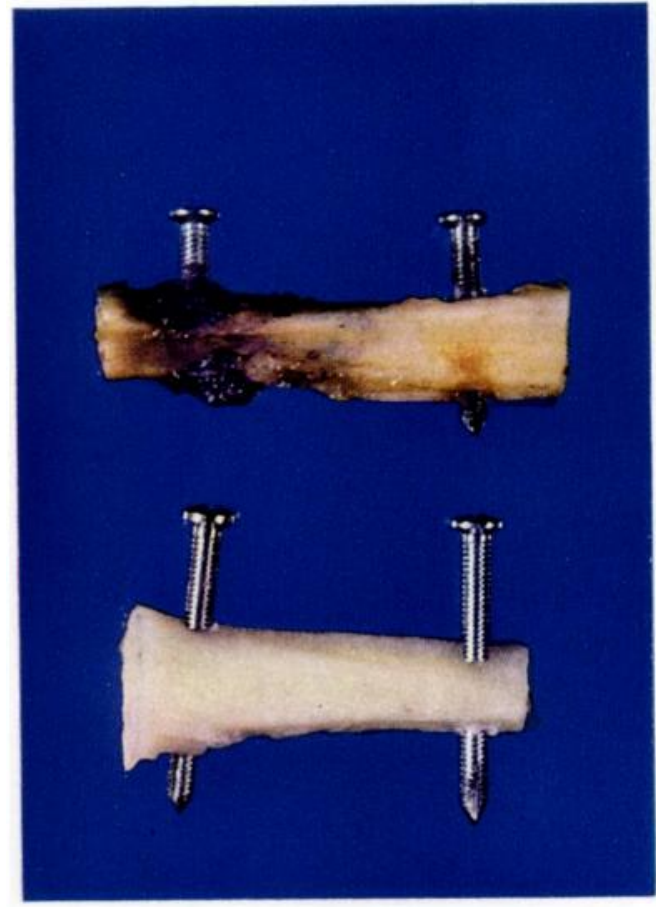

Fig. 7 types. One of these, designated $18 / 8$, contains approximately 18 per cent of chromium and 8 per cent of nickel. The addition of about 2 per cent of molybdenum to such a steel gives an increased resistance to corrosion in the presence of chloride ions and organic acidsas occurs in the tissues of the body. Venable and Stuck have stated the composition limits of a satisfactory stainless steel for use in the body as follows: chromium 17 to 20 per cent; nickel 10 to 14 per cent; molybdenum 2 to 4 per cent; sulphur 0.03 per cent maximum; iron to 100 per cent. Such a material is designated Number 317 by the American Iron and Steel Institute, and is referred to by Venable and Stuck as $18 / 8$ S.Mo.

The screws made in Great Britain for orthopaedic surgery do not always conform to these standards. Examination of a nut, screw and washer of approximately 4.BA size of the so-called Sherman pattern showed: chromium 20.5 per cent; nickel 9.5 per cent; sulphur more than 1 per cent; molybdenum nil. Photograph of the screws shown in Figure 6. Spectrographic examination indicated that nut, screw and washer were all made from material of an identical chemical composition, although, as will be shown later, the three components were not in the same metallurgical condition. The absence of molybdenum and the excess of sulphur (more than three times the permitted maximum) should be noted. It appears, therefore, that the stainless steel supplied for orthopaedic use in Great Britain can differ from that recommended by Venable and Stuck.

Metallographic examination of a polished section of one of these screws showed the presence of many small isolated crystals embedded in the mass of the iron-chromium-nickel alloy (Fig. 8). The crystals consist of metallic sulphides and are hard and brittle. The presence of such crystals of hard compound embedded in the relatively soft iron-chromium-nickel alloy makes the cutting of screw threads much easier, and such a material is called "free cutting." Since these crystals are uniformly distributed throughout the alloy, a voltaic cell may be set up between the crystalline compound and the mass of alloy itself, if the material is in contact with an electrolyte, as they act as two electro-chemically different materials. "Free cutting" stainless steel would be expected to show inferior corrosion resistance to a 
steel consisting of one type of material. It is noteworthy that we did not find these crystals in a different screw of the "wood screw" type.

The effect of surface abrasion-The resistance of stainless steel to corrosion depends upon the presence of a thin, invisible but continuous film of chromium oxide which covers the surface and isolates the metal from a corrosive electrolyte. Screws and plates for orthopaedic use are usually electropolished to produce a smooth surface, and then "passivated" in nitric acid to increase the effectiveness of the oxide film. This film is some $10^{-5}$ millimetres thick and can be easily destroyed by mechanical abrasion. Such abrasion readily occurs at the edge of a hole in a plate by contact with a rotating drill; on the head of the screw by mechanical damage from a screwdriver; at the points of contact between a screw and an edge of the plate, and especially when the male and female threaded portions of a nut and bolt are fastened tightly together. Such mechanical abrasion removes the insulating film, and electrolytic corrosion can begin.

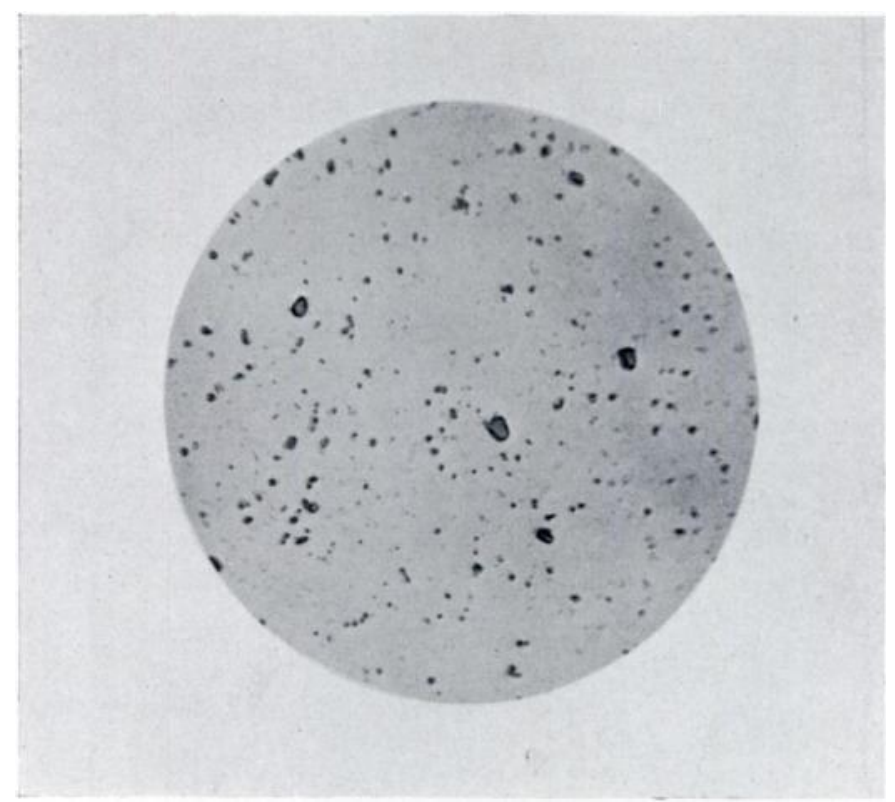

Fig. 8

Polished section of screw showing isolated crystals of metallic sulphides in iron-chromium-nickel alloy.

The "cold weld" effect-Bowden, Williamson and Laing (1954) demonstrated another mechanism by which electrolysis may be produced around and on screws and plates of stainless steel. When a screwdriver is placed in firm contact with a screw, as it must be when the screw is driven home, an actual transfer of metal occurs from the screwdriver to the screw, and vice versa. Another similar transfer occurs when a clamp is applied tightly to a plate, or when any two metals are forced together and later separated. Microscopically the surfaces of these articles are not smooth, but irregular. When two irregular surfaces are clamped together, "high spots" occur where salient points meet, and the pressure at these points produces an actual welding together of the metals (a "cold weld"). When the metal surfaces are later separated, they may fracture apart on either side of some or all of these points, thus transferring metal from the screwdriver or clamp to the screw or plate (Fig. 9). There is no doubt that this can occur. Bowden, Williamson and Laing (1954) showed by radiochemical studies that up to $65 \mu$-grammes of anodic material can be transferred from a screwdriver to a screw. The importance of this is doubtful, but its existence is certain. 

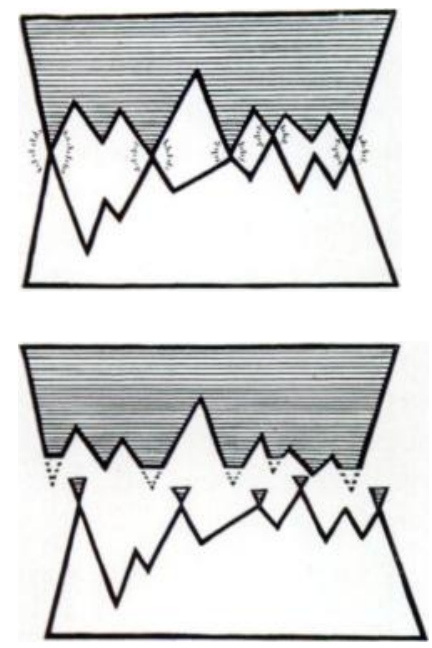

FIG. 9

"Cold weld" effect.

The effect of mechanical distortion-If a piece of almost any common metal is distorted mechanically the distorted portion tends to become anodic with respect to the undistorted portion. This is due to the greater ease with which atoms from an irregular (distorted) lattice arrangement can dissolve than can those of a regular (undistorted) lattice. Such distortion cannot fail to occur during the process of cutting a screw thread, since the process is essentially one of tearing a furrow in a piece of metal.

In stainless steels of the $18 / 8$ type mechanical distortion may also actually change the type of crystal lattice on which the metallic atoms are situated. The 18/8 type of steel exists as a face-centred cubic crystal, an arrangement as shown in Figure 10. This crystal is non-magnetic and is called "austenite." Mechanical distortion may change this nonmagnetic austenite to a magnetic crystal type termed "martensite." The amount of the magnetic modification increases with the degree of mechanical distortion. This magnetic modification of the iron-chromium-nickel alloy (" martensite") has a crystal structure in which the atoms are arranged in a body-centred cubic pattern (Fig. 11).

When this effect is related to the nut, screw and washer already mentioned (free cutting steel containing 20.5 per cent chromium and 9.5 per cent nickel) the washer showed no magnetic response to the hand magnet, the nut showed a weak magnetic response if the magnet

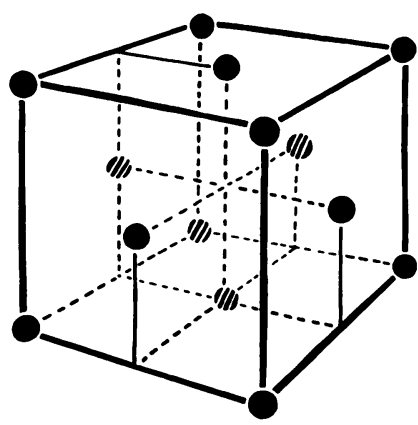

Fig. 10

Face-centred cubic crystal.

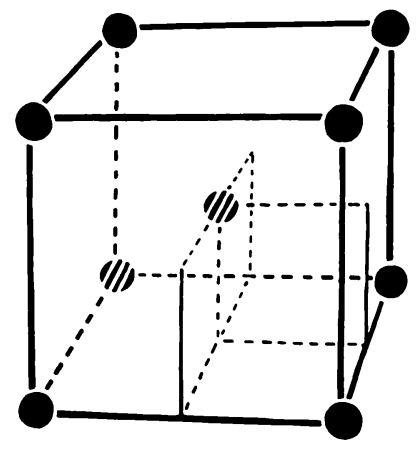

Fig. 11

Body-centred cubic crystal.

was placed near to the screw threaded hole, and the screw itself showed a strong magnetic response at the threaded portion, though not at the head.

This illustrates how mechanical distortion in the process of cutting a screw thread may put chemically similar articles into different metallurgical conditions though originally they were fashioned from the same block of metal. The electro-chemical effect is the same as though two different alloys were present, and a voltaic cell can thus be produced. Mechanical distortion may also occur of course during the insertion of a screw, with similar changes.

The susceptibility of 18/8 type of stainless steel to this effect depends upon the proportion of nickel to chromium. The role of chromium is to provide resistance to corrosion by the formation of a continuous and coherent film of chromium oxide, whereas that of nickel is to produce an alloy in which only one crystal type (austenite) is present. As the chromium content is increased above 18 per cent progressively greater amounts of nickel are required to retain the austenite. When the chromium content is 20.5 per cent (as found in the analysed 
screw) it is desirable to increase the nickel content to about 14 per cent $(9.5$ per cent in this screw).

Differential oxygenation-It must be remembered that an electric current can be set up between two parts of the same metal if one part is exposed to a greater concentration of oxygen in the surrounding electrolyte solution. The part that is more highly oxygenated will become cathodic, with consequent anodic dissolution or corrosion of the other piece. Differential oxygenation can occur in two ways in vivo. First, one screw may lie in scar tissue and another in granulation tissue, tissues which may differ in oxygen potential. Second, a crevice may be formed between two pieces of the same metal in contact with one another. It then becomes more difficult for oxygen to diffuse into the crevice than for it to reach the metal surface. The crevice thus becomes anodic, and tends to corrode.

\section{MISCELLANEOUS FACTORS}

Other factors which may well play a minor but contributory role in producing electrolysis exist. Small temperature changes between one end of the plate-screw system and the other can be shown in vitro to set up voltage changes between these points. Such temperature differences are possible in the body between subcutaneous plates and more deeply buried screws.

\section{CORROSION IN PRACTICE}

It is debatable whether " electrolytic inflammation " can interfere greatly with the healing of the internally fixed fracture. It is our experience that such " inflammation " usually occurs at some distance from the fracture site-for example, around one of the screws-and is

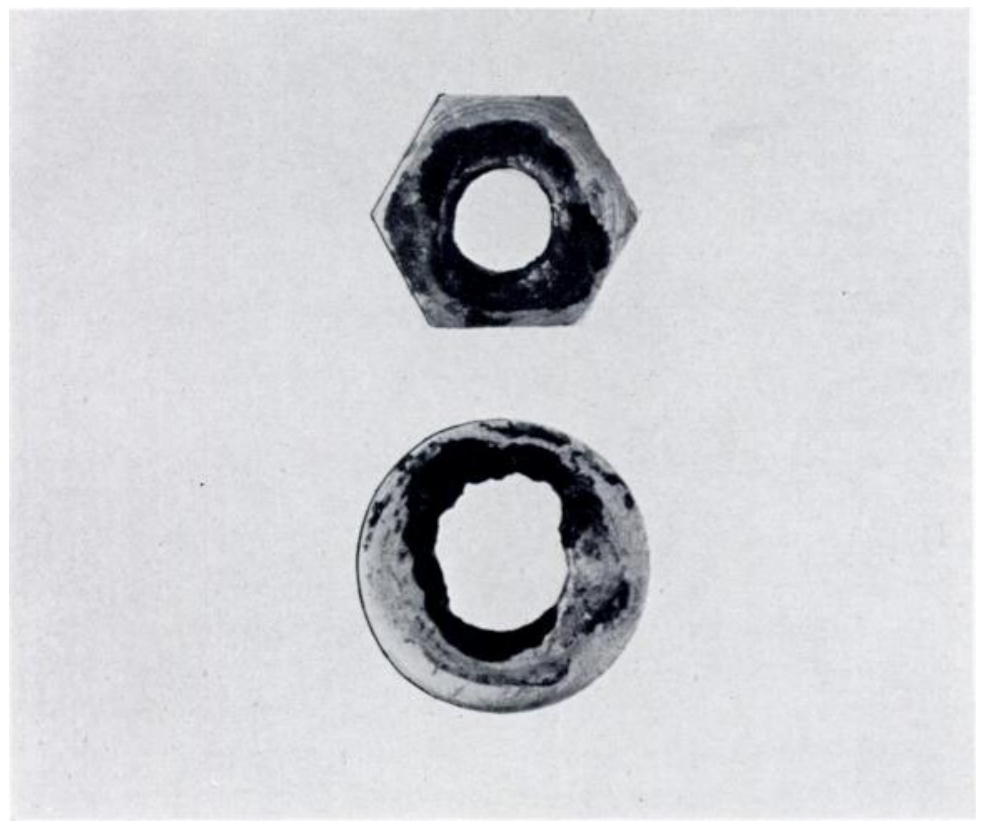

Fig. 12

Nut and washer showing marked corrosion of internal parts.

walled off by fibrous tissue. Here a "chemical abscess" is probably formed and, especially if the plate is subcutaneous, this abscess may point through the skin and become secondarily infected. We have not infrequently seen primary skin healing of the plated fracture without any evidence of sepsis, but with localised wound breakdown six or eight weeks later, which may be caused in this way. Such "chemical abscesses," even without secondary infection, 
eventually lead to the loosening of the screw and loss of fixation. Yet not all screws loosen; indeed some defy attempts at removal. But all screws loosen if the fracture fails to unite. What part of this loosening is electrolytic and what part is due to mechanical stresses in any particular instance is impossible to assess, but it would appear that loose screws can lead to non-union and non-union to loose screws.

Chemical differences between individual screws and between screws and plates certainly exist. These differences become particularly important if it is the practice of the theatre sister to place plates and screws in a common box, for these plates and screws from different batches, or indeed from different manufacturers, may be selected for use in one particular case. They may differ considerably on chemical analysis, with resultant voltaic action.

Even when chemically identical materials are used they may not be in the same metallurgical condition. The threaded portion of a screw or nut may, by mechanical distortion, have been rendered anodic. When they are bolted together other factors will operate to concentrate the amount of corrosion at their points of contact. These factors are the destruction of the protective oxide film by the mechanical abrasion of one thread upon another, and the differential oxygenation effect which tends to make any crevice between them anodic. Corrosion at this point may rapidly destroy the rigidity of fixation. Indeed, an instance has been encountered in which the internally threaded part of a nut has been completely dissolved away (Fig. 12). It seems, therefore, that wherever possible, bolted structures should be avoided, as corrosion occurs at the very point where the mechanical strain is greatest.

A similar but less pronounced effect is obtained between the region of contact of the head and neck of the screw and the plate. Bony dissolution in this region readily causes loss of fixation. Electrolytic changes at this site are much more important than those occurring around the slot of the screw.

\section{THE PREVENTION OF ELECTROLYTIC CORROSION}

Clearly the most important points in the prevention of electrolytic corrosion are the use of identical metals in exactly the same metallurgical condition, and the careful handling of these metals both before and during use, to avoid abrasion of their surfaces and their mechanical distortion.

We insist on a rigid control of the purity of the drugs we use. This control must be extended to the chemical and mechanical standards in the manufacture of orthopaedic materials. (In the course of this study we have noted also marked mechanical differences in supposedly identical materials.)

Ideally, all metals used in the fixation of a fracture, and all metals coming into electrical contact (which implies mechanical contact) should be chemically and metallurgically identical. The clamps and the screwdrivers should be uniform in composition with the screws and the plates. This is a counsel of perfection, but perfection is a worthy aim.

There is little prospect at present of a completely inert substance of sufficient strength for use in the body. No known plastic will take the necessary strains without breaking, even if one uses a steel core within the plastic article. We have tried to reduce corrosion by coating the plates and screws with silicones, which provide an electrically insulating coating, but unhappily this easily chips off during the insertion of the screws, and thus fails to achieve its object.

It is important to remember that dissimilar metals will only act as a voltaic cell if they are in electrical contact at some point. It might be worth while considering the insertion of a thin insulating washer between the plates and the heads of the screws. We have at present no experience of this, but it is noteworthy that we have rarely observed corrosion in the isolated screws holding a bone graft. 


\section{SUMMARY AND CONCLUSIONS}

1. The factors producing electrolytic corrosion of stainless steels are reviewed, and it is shown how several factors operating together can accentuate corrosion in certain positions in the plated fracture.

2. These factors can be minimised by good metallurgy and good engineering on the part of the manufacturers.

3. When and if materials become metallurgically satisfactory, or a truly inert substitute for metal is discovered, it will be possible to assess the true contribution of infection, faulty operative technique and mechanical factors to the failures of internal fixation. Such a critical assessment of these probably more important factors is at present bedevilled by uncertainty as to the purely physical condition of the materials as supplied to the surgeon.

We would like to thank Mr D. Ll. Griffiths, the Director of the University Department of Orthopaedic Surgery, in which this work has been carried out, for his interest and advice; also the Departments of Medical Illustration and Pathology for their photography.

\section{REFERENCES}

Bowden, F. B., Williamson, J. B. P., and Laing, P. G. (1954): Metallic Transfer in Screwing and its Significance in Bone Surgery. Nature, 173, 520.

Venable, C. S., and Stuck, W. G. (1947): The Internal Fixation of Fractures. Oxford: Blackwell Scientific Publications Ltd. 